\title{
Dermatology
}

Dermatology 2008;217:121-123

DOI: $10.1159 / 000131170$

\section{Amicrobial Pustulosis of the Folds Affecting a Young Male without Any Accompanying Autoimmune Diseases}

\section{Ryuhei Okuyama, Takashi Masu, Naka Kumasaka, Setsuya Aiba, Hachiro Tagami}

Department of Dermatology, Tohoku University Graduate School of Medicine, Sendai, Japan

Key Words

Amicrobial pustulosis $\cdot$ Immune abnormality $\cdot$ Autoimmune disease $\cdot$ Neutrophilic cutaneous lupus erythematosus

Amicrobial pustulosis of the folds (APF) is a recurrent, aseptic pyoderma predominantly involving the scalp and cutaneous folds including the external acoustic meatus, pubic region and digits of young females $[1,2]$. Patients exhibit a wide spectrum of autoimmune abnormalities such as elevated serum autoantibodies alone or even autoimmune diseases such as systemic lupus erythematosus (SLE). Almost all the previously reported cases were observed in females. Only 1 male case documented so far suffered from IgA nephropathy and Sjögren's syndrome [3]. We describe here another male patient with APF who had aseptic pustules only on the scalp and retroauricular folds but not in other skin folds. In addition, he showed only positive antinuclear antibodies without any accompanying autoimmune diseases.

\section{Case Report}

A 17-year-old Japanese high school boy visited a nearby hospital with a 1.5-year history of nonpruritic eruptions affecting the scalp and forehead. On his visit, there were multiple scattered pustules on the scalp. He was treated with oral minocycline $200 \mathrm{mg} /$ day, colchicine $30 \mathrm{mg} /$ day, zinc sulfate $360 \mathrm{mg} /$ day or biotin $8 \mathrm{mg} /$ day successively for at least 1 week, but all treatments failed to improve his skin eruptions. He was then referred to us. Examination showed numerous pustules, some of which coalesced forming crusting erythematous plaques on the scalp, forehead and retroauricular folds (fig. 1a). On the other hand, there were no skin changes in the axillae and inguinal folds. Subcutaneous lymph nodes were palpable in the bilateral postauricular and occipital regions (fig. 1b). He had no history of allergic disorders or psoriasis.

The authors have no conflicting financial interests.
Routine laboratory examinations showed an increased white blood cell count of $17,600 / \mathrm{mm}^{3}$ (neutrophils: $74 \%$, lymphocytes: $16 \%$, monocytes: $8 \%$, eosinophils: $2 \%$, basophils: $1 \%$ ) and elevated C-reactive protein of $5.9 \mathrm{mg} / \mathrm{dl}$ (normal range, $0-0.39$ ). Liver and renal function tests were normal. Serum IgE was increased (830 IU; normal range, 0-400). A speckled pattern of antinuclear antibody was detected at 1:2,560 (normal range $<1: 40$ ) without antibodies to ssDNA, dsDNA, Sm, SS-A/Ro or SS-B/La. The plasma zinc levels were normal. There were no symptoms suggestive of collagen diseases. Bacterial cultures from the pustules isolated only a small number of colonies of Staphylococcus epidermidis on several occasions, indicating a secondary colonization.
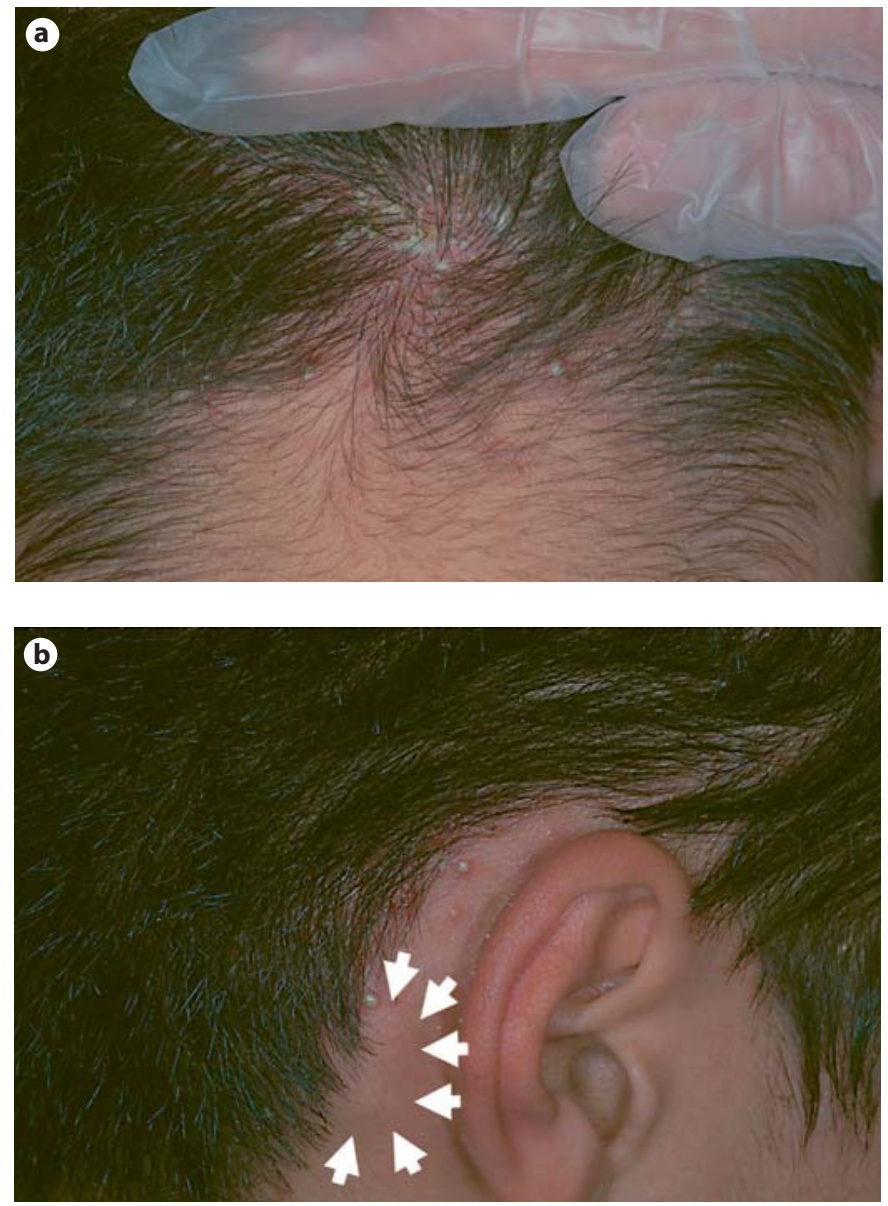

Fig. 1. Clinical features. a Multiple pustules with coalescence arising on the erythematous skin and forming large crusted plaques in the scalp. b Enlarged lymph node in the postauricular region (arrows).

\section{KARGER}

(C) 2008 S. Karger AG, Basel

Fax +41 613061234 E-Mail karger@karger.ch www.karger.com 

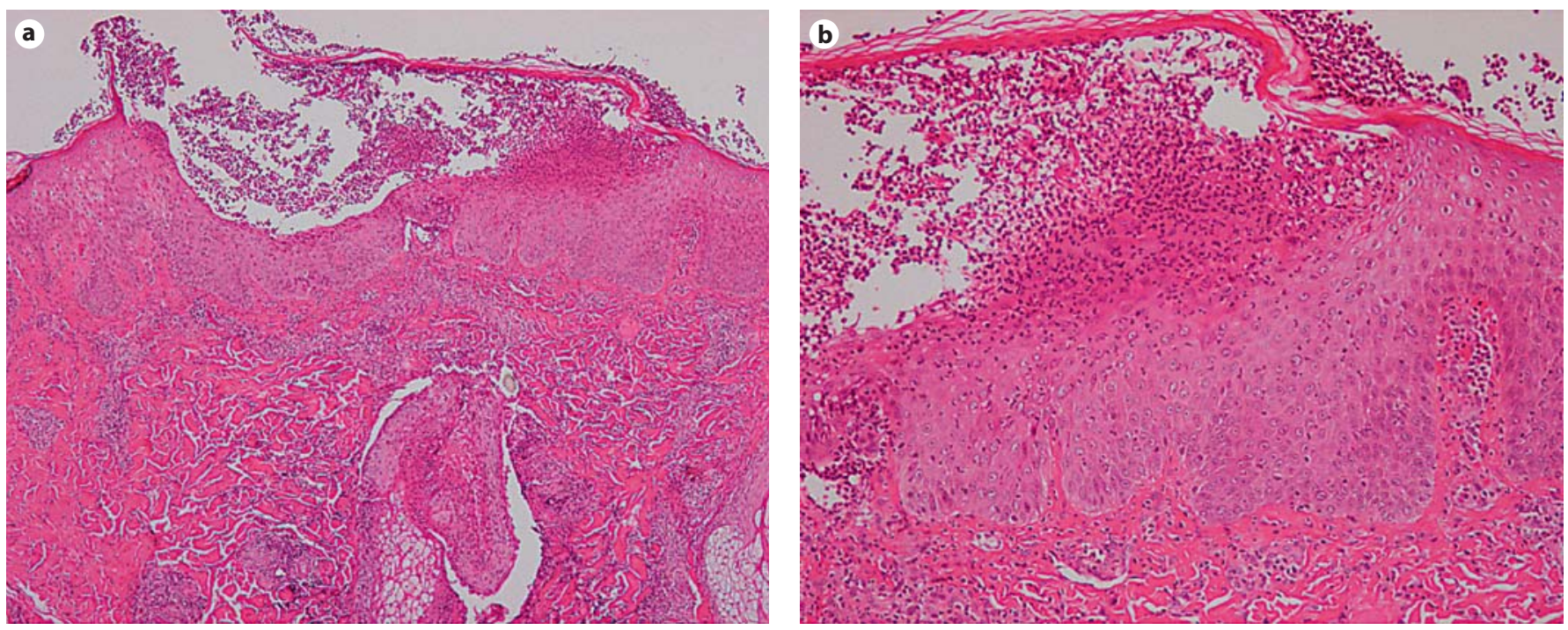

Fig. 2. Histopathological features. a Biopsied specimen of pustule from the scalp showed massive neutrophil infiltration in the epidermis and dermis. Original magnification $\times 40$. b A subcorneal spongiform abscess was observed. Original magnification $\times 100$. c Neutrophils infiltrated around the blood vessels as well as the hair follicles and eccrine ducts. Hematoxylin-eosin stain. Original magnification $\times 100$.

Skin biopsy from the scalp showed an intense dermal infiltration of neutrophils and lymphocytes around the vessels as well as hair follicles and eccrine ducts, extending to the epidermis. There was a dense neutrophilic infiltration in the epidermis forming a subcorneal spongiform pustule without liquefaction degeneration of the basal layer (fig. 2). In the dermis, these cellular infiltrations were associated with nuclear dust scattered between collagen fibers. On direct immunofluorescence, there was no deposition of IgG, IgA, IgM or complements.

He was treated with oral prednisone, $20 \mathrm{mg}$ daily, and topical clobetasol propionate $0.05 \%$ ointment for 4 weeks. The patient's response was variable, with improvement seen on some visits but worsening noted on others. Then, instead of corticosteroids, we administered roxithromycin $300 \mathrm{mg}$ daily for 8 weeks, which has immunomodulatory properties due to its inhibitory effect on neutrophil infiltration $[4,5]$. However, it failed to alleviate his eruptions. He did not request any further treatment, turning

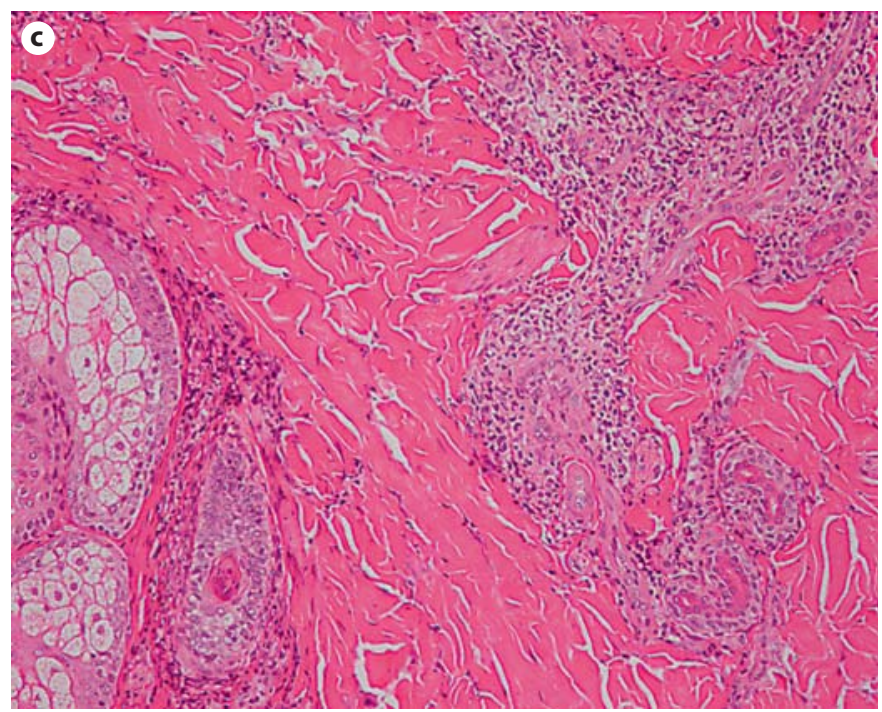

down our suggestion to try dapsone or cyclosporine because of fear of their side effects. A follow-up made 3 months later demonstrated no special change in the clinical appearance of the scalp pustules.

\section{Discussion}

APF is a rare clinical entity. The clinical features are relapsing, aseptic, pustular eruptions, mainly affecting the scalp and cutaneous folds. Typical histological features are intraepidermal spongiform pustules with a neutrophilic infiltrate in the dermis. The etiology of amicrobial pustulosis is unclear. However, it is noteworthy that almost all the previously reported cases with amicrobial pustulosis were found in females showing various autoantibodies. Despite their immunological abnormalities most of them did not necessarily meet the diagnostic criteria for particular autoimmune diseases. Among them, SLE that was noted in 3 cases [6] was the most frequently observed disorder. The female predominance 
may be explained by the underlying immunological disorders, such as SLE, which show a similar female prevalence.

APF is characterized by neutrophilic infiltration that usually constitutes the first-line defense against external insults. Its pathologic condition can be classified as autoinflammation disease in contrast to SLE that is considered as a typical example of autoimmune disease, which are characterized by specific $\mathrm{T}$ cells or pathogenic antibodies. However, SLE is sometimes reported in association with aseptic neutrophilic dermatoses such as Sweet's disease, subcorneal pustulosis or pemphigus foliaceus [7]. Furthermore, the neutrophilic infiltration can become predominant in some variants of LE, for example bullous LE or urticarial vasculitis. The association of LE with APF and other neutrophilic dermatoses seems to indicate that the pathogenesis of LE includes not only autoimmune but also autoinflammatory pathways. Based on this, Lipsker and Saurat [8] recently proposed the new concept of 'neutrophilic cutaneous lupus erythematosus'. In these conditions, it has been suggested that immune complexes activate complements in the skin to generate chemotactic factors that induce neutrophilic infiltration. However, direct immunofluorescence did not demonstrate any deposition of immune complexes in the skin in our case or in others $[3,6,9,10]$. Thus, the relationship between the neutrophilic infiltration and the underlying autoimmune disease remained poorly understood.

APF is characterized by the aseptic pustules localized to major skin folds and the scalp. Recently Marzano et al. [2, 6] summarized 21 previous cases which showed that lesions were present in at least one of the major skin folds, such as axillae or groins, or in one of the minor skin folds, such as retroauricular folds or external canals in all cases. Furthermore, the anogenital area was always involved in addition to the scalp affected in 20 of 22 cases. Based on the analysis, they proposed diagnostic criteria for APF which include pustulosis distributed to the major fold, minor folds and anogenital area as an obligate requirement. From such a clinical view point our present case is unique in that pustules were localized only to the scalp and retroauricular folds but spared the major skin folds or anogenital region, which did not satisfy the above-mentioned criteria. We cannot exclude the possibility that our case was not consistent with APF. Multiple pustules in the scalp may develop in several diseases (table 1), but our case did not match those noted in them. Although our case may represent a new pattern among these neutrophilic dermatoses, we rather consider that the incomplete presence of APF features may be related to the fact that it was observed in a young male. The occurrence in males may further explain the fact that there has not been any accompanying autoimmune disease in this case despite the presence of antinuclear antibody that showed a speckled pattern. Since some cases were reported to develop autoimmune diseases after the onset of APF, he should be carefully followed up for the development of collagen diseases in the future.

The therapy for amicrobial pustulosis is not well standardized. Systemic corticosteroids have been employed with good results [1, $3,10,11]$ but are not always effective [12]. Potent topical corticosteroids may occasionally be helpful $[1,13]$. In addition, chloroquine [11], dapsone [10], cyclosporine [10], colchicines [9], cimetidine [2] and zinc [12] have been used for its therapy. Although the symptoms were not severe in our case, he did not respond satisfactorily to any of the above therapeutic modalities. Recently, Marzano et al. [6] reported that a combination of cimetidine and ascorbic acid was applied to induce clinical remission.
Table 1. Differential diagnosis of pustulosis on the scalp

Amicrobial pustulosis of the folds

Pustular psoriasis

Subcorneal pustular dermatosis

Superficial granulomatous pyoderma

Erosive pustular dermatosis of the scalp

Seborrheic dermatitis

Acne vulgaris

Dissecting folliculitis/folliculitis decalvans

Tinea capitis/kerion celsi

Eccrine hidradenitis

\section{References}

1 Lagrange S, Chosidow O, Piette JC, Wechsler B, Godeau P, Frances C: A peculiar form of amicrobial pustulosis of the folds associated with systemic lupus erythematosus and other auto-immune diseases. Lupus 1997;6:514-520.

2 Marzano AV, Capsoni F, Berti E, Gasparini G, Bottelli S, Caputo R: Amicrobial pustular dermatosis of cutaneous folds associated with autoimmune disorders: a new entity? Dermatology 1996;193:88-93.

-3 Natsuga K, Sawamura D, Homma E, Nomura T, Abe M, Muramatsu R, Mochizuki T, Koike T, Shimizu H: Amicrobial pustulosis associated with IgA nephropathy and Sjögren's syndrome. J Am Acad Dermatol 2007:57:523-526.

4 Siddiqui J: Immunomodulatory effects of macrolides: implications for practicing clinicians. Am J Med 2004;117(suppl 9A):26S-29S.

5 Tamaoki J, Kadota J, Takizawa H: Clinical implications of the immunomodulatory effects of macrolides. Am J Med 2004;117(suppl 9A):5S$11 \mathrm{~S}$

6 Marzano AV, Ramoni S, Caputo R: Amicrobial pustulosis of the folds: report of 6 cases and a literature review. Dermatology 2008;216:305311.

7 Callen JP: Neutrophilic dermatoses. Dermatol Clin 2002;20:409-419.

-8 Lipsker D, Saurat J-H: Neutrophilic cutaneous lupus erythematosus: at the edge between innate and acquired immunity. Dermatology 2008; 216:283-286.

$\checkmark 9$ Kerl K, Masouye I, Lesavre P, Saurat JH, Borradori L: A case of amicrobial pustulosis of the folds associated with neutrophilic gastrointestinal involvement in systemic lupus erythematosus. Dermatology 2005;211: 356-359.

10 Kuyama M, Fujimoto W, Kambara H, Egusa M, Saitoh M, Yamasaki O, Maehara K, Ohara A, Arata J, Iwatsuki K: Amicrobial pustular dermatosis in two patients with immunological abnormalities. Clin Exp Dermatol 2002;27:286-289.

11 Stefanidou MP, Kanavaros PE, Stefanaki KS, Tosca AD: Amicrobial pustulosis of the folds: a cutaneous manifestation associated with connective tissue disease. Dermatology 1998;197:394-396.

- 12 Beneton N, Wolkenstein P, Bagot M, Cosnes A, Wechsler J, Roujeau JC, Revuz J: Amicrobial pustulosis associated with autoimmune diseases: healing with zinc supplementation. Br J Dermatol 2000;143:13061310 .

13 Inui S, Azukizawa H, Asada H, Itami S: Amicrobial pustulosis with antinuclear antibodies and rheumatoid factor. Br J Dermatol 2006;154: $568-569$.

Ryuhei Okuyama

Department of Dermatology

Tohoku University Graduate School of Medicine

1-1 Seiryo-machi, Aoba-ku, Sendai 980-8574 (Japan)

Tel. +81 22717 7271, Fax +81 227177361

E-Mail rokuyama@mail.tains.tohoku.ac.jp 